\author{
MITSUBISHI ELECTRIC RESEARCH LABORATORIES \\ http://www.merl.com
}

\title{
Lyapunov-Based Control of the Sway Dynamics for Elevator Ropes with Time-Varying Lengths
}

\author{
Benosman, $\mathrm{M}$.
}

TR2014-077 August 2014

\begin{abstract}
In this work we study the problem of rope sway dynamics control for elevator systems, with timevarying rope lengths. We formulate this problem as a nonlinear control problem and propose nonlinear controllers based on Lyapunov theory for time-varying systems. We study the stability of the proposed controllers, and test their performances on a numerical example.
\end{abstract}

IFAC 2014

This work may not be copied or reproduced in whole or in part for any commercial purpose. Permission to copy in whole or in part without payment of fee is granted for nonprofit educational and research purposes provided that all such whole or partial copies include the following: a notice that such copying is by permission of Mitsubishi Electric Research Laboratories, Inc.; an acknowledgment of the authors and individual contributions to the work; and all applicable portions of the copyright notice. Copying, reproduction, or republishing for any other purpose shall require a license with payment of fee to Mitsubishi Electric Research Laboratories, Inc. All rights reserved. 



\title{
Lyapunov-Based Control of the Sway Dynamics for Elevator Ropes with Time-Varying Lengths
}

\author{
M. Benosman* \\ * Mitsubishi Electric Research Laboratories, 201 Broadway Street, \\ Cambridge, MA 02139, USA (benosman@merl.com)
}

\begin{abstract}
In this work we study the problem of rope sway dynamics control for elevator systems, with time-varying rope lengths. We formulate this problem as a nonlinear control problem and propose nonlinear controllers based on Lyapunov theory for time-varying systems. We study the stability of the proposed controllers, and test their performances on a numerical example.
\end{abstract}

\section{INTRODUCTION}

Fast and high performance elevator systems are becoming a necessity in this world of high-rise buildings. This rises the question of safety in elevator systems. One of the common safety problems is the rope sway effect. Indeed, even slight external disturbances on a high-rise building, e.g. wind gust or earthquake, can lead to large rope sway within the elevator shaft. Considering the length of the ropes and their heavy weight, it is clear that the rope sway can damage the equipments that are installed in the elevator shaft and can also cause damages to the elevator shaft structure itself, without mentioning the potential danger caused for the elevator passengers. For these reasons, it is very important to be able to control the rope dynamics within the elevator shaft. Furthermore, due to cost constraints, it is preferable to be able to do so, with minimum actuation capabilities. Several papers have been dedicated to the problem of modelling and control of long elevator ropes, e.g. Kaczmarczyk [2011], Kaczmarczyk et al. [2009], W.D.Zhu and Xu [2003], W.D.Zhu and Teppo 2003], W.D.Zhu and Chen [2006], Fujino et al. [1993]. In Fujino et al. [1993], a simple model of a cable attached to an actuator at its free end is used to investigate the stiffening effect of the control force on the cable. An energy analysis is used to tune an open-loop sinusoidal force applied to the cable. In W.D.Zhu and Teppo [2003], a scaled model for high-rise, high-speed elevators is developed. The model is used to analyze the influence of the car motion profiles on the lateral vibrations of the elevator cables. An active stiffness control of the transverse vibrations of elevator ropes is presented in Kaczmarczyk [2011]. The author propose a nonlinear modal feedback to drive an actuator pulling on one end of the rope. The control performance is investigated by numerical tests. In W.D.Zhu and Chen [2006], the authors proposed a novel idea to dissipate the transversal energy of an elevator rope. The authors used a passive damper attached between the car and the rope. Numerical analysis of the transverse motion average energy was conducted to find the optimal value of the damper coefficient. In Benosman and Fukui [2014], the authors presented preliminary results on nonlinear Lyapunov control of the elevators rope sway problem, but only the case of static elevators, i.e., constant rope lengths, was studied there.

In this work we propose to extend the results of Benosman and Fukui [2014] to the case of time-varying rope lengths, i.e., controlling the rope sway when the elevator car is moving, which is a more challenging problem, due to the fact that the closed-loop model becomes in this case a time-varying nonlinear system. Similarly to Benosman and Fukui [2014], we use an active actuator to pull on oneside of the ropes. We show that in this case the model of the elevator rope together with its actuator writes as a nonlinear time-varying model, which we use to develop nonlinear Lyapunov-based feedback controllers to stabilize the rope sway dynamics. We study the stability of the closed-loop dynamics, and show the performances of these controllers on a numerical example.

The paper is organized as follows: We start the paper with some preliminaries in Section 2. In Section 3, we recall the model of the system. Next, in Section 4, we present the main results of this work, namely, the nonlinear Lyapunovbased controllers, together with their stability analysis. Section 5 is dedicated to some numerical results. Finally, we conclude the paper with a brief summary of the results in Section 6.

\section{NOTATIONS AND PRELIMINARIES}

Throughout the paper, $\mathbb{R}, \mathbb{R}_{+}$denotes the set of real, and the set of nonnegative real numbers, respectively. For $x \in \mathbb{R}^{N}$ we define $|x|=\sqrt{x^{T} x}$, we denote by $A_{i j}, i=$ $1, \ldots, n, j=1, \ldots, m$ the elements of the matrix $A$, and denote by $\operatorname{sgn}($.$) the signum function.$

\section{ELEVATOR ROPE MODELLING}

In this section we first recall the infinite dimension model, i.e., partial differential equation (PDE), of a moving hoist cable, with non-homogenous boundary conditions. Secondly, to be able to reduce the PDE model to an ODE model using a Galerkin reduction method, we introduce a change of variables and re-write the first PDE model in a new coordinates, where the new PDE model has zero boundary conditions. Let us first enumerate the assumptions under which our model is valid.

- The elevator ropes are modelled within the framework of string theory.

- The elevator car is modelled as a point mass.

- The vibration in the second lateral direction is not included.

- The suspension of the car against its guide rails is assumed to be rigid. Under the previous assumption, fol- 


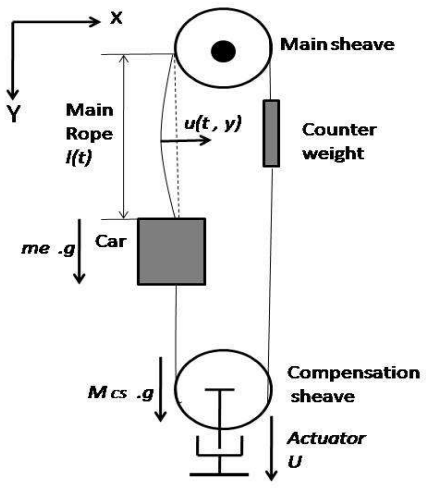

Fig. 1. Schematic representation of an elevator shaft showing the different variables used in the model

lowing W.D.Zhu and Xu [2003], Kaczmarczyk [2011], the general PDE model of an elevator rope, depicted on Figure 1 , is given by

$$
\begin{aligned}
& \rho\left(\frac{\partial^{2}}{\partial t^{2}}+v^{2}(t) \frac{\partial^{2}}{\partial y^{2}}+2 v(t) \frac{\partial}{\partial y \partial t}+a \frac{\partial}{\partial y}\right) u(y, t) \\
& -\frac{\partial}{\partial y} T(y, t) \frac{\partial u(y, t)}{\partial y}+c_{p}\left(\frac{\partial}{\partial t}+v(t) \frac{\partial}{\partial y}\right) u(y, t)=0
\end{aligned}
$$

where $u(y, t)$ is the lateral displacement of the rope. $\rho$ is the mass of the rope per unit length. $T$ is the tension in the rope, which varies depending on which rope in the elevator system we are modelling, i.e., main rope, compensation rope, etc. $c_{p}$ is the damping coefficient of the rope per unit length. $v=\frac{\partial l(t)}{\partial t}$ is the elevator rope velocity, where $l: \mathbb{R} \rightarrow \mathbb{R}$ is a function (at least $C^{2}$ ) modelling the time-varying rope length. $a=\frac{\partial^{2} l(t)}{\partial t^{2}}$ is the elevator rope acceleration.

The PDE (1) is associated with the following two boundary conditions:

$$
\begin{aligned}
& u(0, t)=f_{1}(t) \\
& u(l(t), t)=f_{2}(t)
\end{aligned}
$$

where $f_{1}(t)$ is the time varying disturbance acting on the rope at the level of the machine room, due to external disturbances, e.g. wind gust. $f_{2}(t)$ is the time varying disturbance acting at the level of the car, due to external disturbances. In this work we assume that the two boundary disturbances acting on the rope are related via the relation:

$$
f_{2}(t)=f_{1}(t) \sin \left(\frac{\pi(H-l)}{2 H}\right), H \in \mathbb{R}
$$

where $H$ is the height of the building. This expression is an approximation of the propagation of the boundary disturbance $f_{1}$ along the building structure, based on the length $l$, it leads to $f_{2}=f_{1}$ for a length 0 (which is expected), and a decreasing force along the building until is vanishes at $l=H, f_{2}=0$ (which makes sense, since the effect of any disturbance $f_{1}$, for example wind gusts, is expected to vanish at the bottom of the building). As we mentioned earlier the tension of the rope $T(y)$ depends on the type of the rope that we are dealing with. In the sequel, we concentrate on the main rope of the elevator, the remaining ropes are modelled using the same steps by simply changing the rope tension expression.

For the case of the main rope, the tension is given by

$T(y, t)=\left(m_{e}+\rho(l(t)-y)\right)(g-a(t))+0.5 M_{c s} g+U(t)$ where $g$ is the standard gravity constant, $m_{e}, M_{c s}$ are the mass of the car and the compensating sheave, respectively, and $U(t)$ is the control tension applied by an actuator attached to the compensation sheave (the same actuator placement has been considered in Kaczmarczyk [2011]). Next, we reduce the PDE model (1) to a more tractable model for control, using a projection Galerkin method or assumed mode approach, e.g. Meirovitch [1967].

To be able to apply the assumed mode approach, let us first apply the following one-to-one change of coordinates to the equation (1)

$$
u(y, t)=w(y, t)+\frac{l(t)-y}{l(t)} f_{1}(t)+\frac{y}{l(t)} f_{2}(t)
$$

One can easily see that this change of coordinates implies trivial boundary conditions

$$
\begin{aligned}
& w(0, t)=0 \\
& w(l(t), t)=0
\end{aligned}
$$

After some algebraic and integral manipulations, the PDE model (1) writes in the new coordinates as

$$
\begin{gathered}
\rho \frac{\partial^{2} w}{\partial t^{2}}+2 v(t) \rho \frac{\partial^{2} w}{\partial y \partial t}+\left(\rho v^{2}-T(y, t)\right) \frac{\partial^{2} w}{\partial y^{2}}+G(t) \frac{\partial w}{\partial y} \\
+c_{p} \frac{\partial w}{\partial t}=y\left(-\rho s_{1}(t)-c_{p} s_{2}(t)\right)-\rho f_{1}^{(2)}+s_{4}(t)
\end{gathered}
$$

where $G(t)=\rho a(t)-\frac{\partial T}{\partial y}+c_{p} v(t)$, and the $s_{i}$ variables are defined as

$$
\begin{aligned}
& s_{1}(t)=\frac{l l^{(2)}-2 \dot{l}^{2}}{l^{3}} f_{1}(t)+2 \frac{\dot{l}}{l^{2}} \dot{f}_{1} \\
& +\frac{\left(l^{3} f_{2}^{(2)}-f_{2} l^{2} l^{(2)}+2 l \dot{l}^{2} f_{2}-2 l^{2} i \dot{f}_{2}\right)}{l^{4}}-\frac{f_{1}^{(2)}}{l} \\
& s_{2}(t)=\frac{\dot{l}}{l_{f_{2}}} f_{1}-\frac{\dot{f}_{1}}{l}+\frac{\dot{f}_{2}}{l}-f_{2} \frac{i}{l^{2}} \\
& s_{3}(t)=\frac{f_{1}}{l} \\
& s_{4}(t)=-2 v(t) \rho s_{2}(t)-G(t) s_{3}(t)-c_{p} \dot{f}_{1}(t)
\end{aligned}
$$

associated with the two-point boundary conditions

$$
w(0, t)=0, w(l(t), t)=0 .
$$

Now instead of dealing with the PDE (1) with non-zero boundary conditions, we can use the equivalent model, given by equation (7) associated with trivial boundary conditions (9).

Following the assumed-modes technique, the solution of the equation $(7),(9)$ writes as

$$
w(y, t)=\sum_{j=1}^{j=N} q_{j}(t) \phi_{j}(y, t), \quad N \in \mathbb{N}
$$

where $N$ is the number of bases (modes), included in the discretization, $\phi_{j}, j=1, \ldots, N$ are the discretization bases and $q_{j}, \quad j=1, \ldots, N$ are the discretization coordinates. In order to simplify the analytic manipulation of the equations, the base functions are chosen to satisfy the following normalization constraints

$$
\int_{0}^{l(t)} \phi_{j}^{2}(y, t) d y=1, \int_{0}^{l(t)} \phi_{i}(y, t) \phi_{j}(y, t) d y=0, \quad \forall i \neq j
$$

To further simplify the base functions, we define the normalized variable, e.g. W.D.Zhu and Chen [2006], W.D.Zhu and $\mathrm{Xu}[2003]$

$$
\xi(t)=\frac{y(t)}{l(t)}
$$


and the normalized base functions

$$
\phi_{j}(y, t)=\frac{\psi_{j}(\xi)}{\sqrt{l(t)}}, \quad j=1, \ldots, N
$$

In these new coordinates the normalization constraints (11) write as

$$
\int_{0}^{1} \psi_{j}^{2}(\xi) d \xi=1, \int_{0}^{1} \psi_{i}(\xi) \psi_{j}(\xi) d \xi=0, \quad \forall i \neq j
$$

After classical (e.g. refer to W.D.Zhu and $\mathrm{Xu}$ [2003]) discretization of the PDE-based model (7), (9), we can write the reduced ODE-model based on $N$-modes as

$M \ddot{q}+C(t) \dot{q}+(K(t)+\beta(t) U) q=F(t), q \in \mathbb{R}^{N}, F \in \mathbb{R}^{N}$

where

$$
\begin{aligned}
& M_{i j}=\rho \delta_{i j} \\
& C_{i j}=\rho l^{-1} i\left(2 \int_{0}^{1}(1-\xi) \psi_{i}(\xi) \psi_{j}^{\prime}(\xi) d \xi-\delta_{i j}\right)+c_{p} \delta_{i j} \\
& K_{i j}=\frac{1}{4} \rho l^{-2} i^{2} \delta_{i j}-\rho l^{-2} i^{2} \int_{0}^{1}(1-\xi)^{2} \psi_{i}^{\prime}(\xi) \psi_{j}^{\prime}(\xi) d \xi \\
& +\rho l^{-1}(g-a(t)) \int_{0}^{1}(1-\xi) \psi_{i}^{\prime}(\xi) \psi_{j}^{\prime}(\xi) d \xi+m_{e} l^{-2}(g-a(t)) \int_{0}^{1} \psi_{i}^{\prime}(\xi) \psi_{j}^{\prime}(\xi) d \xi \\
& +\rho\left(l^{-2} i^{2}-l^{-1} \ddot{l}\right)\left(0.5 \delta_{i j}-\int_{0}^{1}(1-\xi) \psi_{i}(\xi) \psi_{j}^{\prime}(\xi) d \xi\right)- \\
& c_{p} l^{-1}\left(\int_{0}^{1} \psi_{i}(\xi) \psi_{j}^{\prime}(\xi) \xi d \xi+0.5 \delta_{i j}\right)+0.5 M_{c s} g l^{-2} \int_{0}^{1} \psi_{i}^{\prime}(\xi) \psi_{j}^{\prime}(\xi) d \xi \\
& \beta_{i i}=l^{-2} \int_{0}^{1} \psi_{i}^{\prime 2} d \xi=l^{-2} \tilde{\beta}_{i i} \\
& \beta_{i j}=\tilde{\beta}_{i j}=0, \forall i \neq j \\
& F_{i}(t)=-l \sqrt{l}\left(\rho s_{1}(t)+c_{p} s_{2}(t)\right) \int_{0}^{1} \psi_{i}(\xi) \xi d \xi \\
& +\sqrt{l}\left(s_{4}(t)-\rho f_{1}^{(2)}(t)\right) \int_{0}^{1} \psi_{i}(\xi) d \xi \\
& \delta_{i j}=\left\{\begin{array}{l}
0, i \neq j \\
1, i=j
\end{array}\right.
\end{aligned}
$$

where $s_{i}, i=1,2,3,4$ are given in (8).

If we use the classical definition of the state vector $z=$ $(q, \dot{q})^{T}$, then it is easy to see that the obtained ODE model is a time-varying bilinear(because of the term $U q$ ) model in the state $z$ and the control vector $U$.

\section{MAIN RESULT: LYAPUNOV-BASED CONTROLLERS}

In this section we present Lyapunov-based feedback controllers designed to stabilize the rope sway dynamics, for the case of time-varying ropes lengths, satisfying the following assumption.

Assumption 1. The time varying length function $l$ : $\mathbb{R}_{+} \rightarrow \mathbb{R}_{+}$is $\mathbb{C}^{2}$, and satisfies: $l(t) \in\left[l_{\text {min }}, l_{\text {max }}\right]$, $\dot{l}(t) \in\left[0, \dot{l}_{\text {max }}\right], \ddot{l}(t) \in\left[0, \ddot{l}_{\text {max }}\right], \quad \forall t \in \mathbb{R}_{+}$, where $l_{\min }, l_{\max }, i_{\max }, \ddot{l}_{\max }$ are given constants.

The first controller deals with the case where the building, hosting a moving elevator, sustains a brief (impulse-like) external disturbance. For example, an earthquake impulse with a sufficient force to make the top of the building oscillate, or a strong wind gust that happens over a short period of time, exciting the building structure and implying residual vibrations of the building even after the wind gust interruption. In these cases, the elevator ropes will vibrate, starting from a non-zero initial conditions, due to the impulse-like external disturbances (i.e., happening over a short time interval), and this case correspond to the model (15), (16) with non-zero initial conditions and zero external disturbances. We can now state the following theorem.

Theorem 1. Consider the rope dynamics (15), (16), with non-zero initial conditions, with no external disturbances, i.e., $f_{1}(t)=f_{2}(t)=0, \forall t$, and with time-varying length $l$ satisfying Assumption 1, then the feedback control

$$
U(z)=u_{\max } \frac{\dot{q}^{T} \tilde{\beta} q|q|^{2}}{\sqrt{1+\left(\dot{q}^{T} \tilde{\beta} q\right)^{2}}}
$$

where $z=\left(q^{T}, \dot{q}^{T}\right)^{T}$, implies that $q(t) \rightarrow 0$, for $t \rightarrow 0$, furthermore $|U|$ decreases with the decrease of $|q|^{2}$.

Proof:

We define the time-varying control Lyapunov function as

$$
V(z, t)=\frac{1}{2} \dot{q}^{T}(t) M \dot{q}(t)+\frac{1}{2} q^{T}(t) K(t) q(t)
$$

where $z=\left(q^{T}, \dot{q}^{T}\right)^{T}$.

First we compute the derivative of the Lyapunov function along the dynamics (15), without disturbances, i.e., $F(t)=$ $0, \forall t$

$$
\begin{gathered}
\dot{V}(z, t)=\dot{q}^{T}(-C \dot{q}-K q-\beta U q)+q^{T} K \dot{q}+\frac{1}{2} q^{T} \dot{K}(t) q \\
=-\dot{q}^{T} C \dot{q}-\dot{q}^{T} \beta q U+\frac{1}{2} q^{T} \dot{K}(t) q
\end{gathered}
$$

Next, based on Assumption 1, we can write

which leads to

$$
\exists c>0, \text { s.t. } \dot{K}(t) \leq c I_{n \times n}, \forall t
$$

$$
\dot{V}(z, t) \leq-\dot{q}^{T} \beta q U+\frac{1}{2} c|q|^{2}
$$

using $U$ defined in (17), we have

$$
\begin{aligned}
& \dot{V}(z, t) \leq-u_{\max } l^{-2} \frac{\left(\dot{q}^{T} \tilde{\beta} q\right)^{2}|q|^{2}}{\sqrt{1+\left(\dot{q}^{T} \tilde{\beta} q\right)^{2}}}+\frac{1}{2} c|q|^{2} \\
& \leq\left(\frac{1}{2} c-u_{\max } l^{-2} \frac{\left(\dot{q}^{T} \tilde{\beta} q\right)^{2}}{\sqrt{1+\left(\dot{q}^{T} \tilde{\beta} q\right)^{2}}}\right)|q|^{2}
\end{aligned}
$$

this shows that $\dot{V}(z, t)$ decreases along (15), as long as, $(q, \dot{q})$ satisfies $\frac{1}{2} c-u_{\max } l^{-2} \frac{\left(\dot{q}^{T} \tilde{\beta} q\right)^{2}}{\sqrt{1+\left(\dot{q}^{T} \tilde{\beta} q\right)^{2}}}<0$, and when $(q, \dot{q})$ enters the set $\left\{\left(q^{T}, \dot{q}^{T}\right)^{T} \in \mathbb{R}^{2 N}\right.$, s.t. $\frac{1}{2} c-$ $\left.u_{\max } l^{-2} \frac{\left(\dot{q}^{T} \tilde{\beta} q\right)^{2}}{\sqrt{1+\left(\dot{q}^{T} \tilde{\beta} q\right)^{2}}} \geq 0\right\}$, it stays in it, which makes $V(z, t)$ bounded.

Next from (21), we can write

$$
\dot{V}(z, t) \leq \frac{1}{2} q^{T} c q
$$

thus

$$
\begin{aligned}
& +\infty>V(z(0), 0)-V(z(t), t) \geq-\frac{1}{2} \int_{0}^{t} q^{T} c q d t \\
& \Rightarrow \int_{0}^{t} q^{T} \text { cqdt is bounded. }
\end{aligned}
$$

Since the boundedness of $V$ implies boundedness of $\dot{q}$, we conclude about the uniform continuity of $q$ and finally using Barbalat's Lemma, e.g. Khalil [1996], we conclude that $\lim _{t \rightarrow+\infty} q(t)=0$. The fact that $|U|$ decreases with the decrease of $|q|^{2}$, is concluded from the upper-bound

$$
|U| \leq u_{\max }|q|^{2}
$$

Next, we present a controller which deals with the case of a moving elevator in a building under sustained external disturbances, e.g. sustained wind forces. In this case $F(t) \neq 0$ over a non-zero time interval, and satisfies the following assumption.

Assumption 2. The time varying disturbance functions $f_{1}, f_{2}$ are such that, the function $F(t)$ is bounded, i.e., $\exists F_{\text {max }}$, s.t. $|F(t)| \leq F_{\text {max }}, \forall t$. 
Under this assumption, we propose the following result.

Theorem 2. Consider the rope dynamics (15), (16), under non-zero external disturbances, i.e., $f_{1}(t) \neq 0, f_{2}(t) \neq 0$ satisfying Assumption 2, and with time-varying length $l$ satisfying Assumption 1, then the feedback control

$$
\begin{aligned}
& U(z)=\frac{u_{\max } \dot{q}^{T} \tilde{\beta} q}{\left.\sqrt{1+\left(\dot{q}^{T} \tilde{\beta} q\right.}\right)^{2}}+k_{1}\left(\dot{q}^{T} \tilde{\beta} q\right)\left(F_{\max }+\epsilon\right)|\dot{q}| \\
& +k_{2}\left(\dot{q}^{T} \tilde{\beta} q\right)|q|^{2} \\
& k_{1}>0, k_{2}>0, \epsilon>0
\end{aligned}
$$

where $z=\left(q^{T}, \dot{q}^{T}\right)^{T}$, ensures that the state vector $x$ converges to the $\omega$-limit set $S_{1}=\left\{\left(q^{T}, \dot{q}^{T}\right)^{T} \in \mathbb{R}^{2 N}\right.$, s.t. $\frac{c}{2}-$ $\left.k_{2} l^{-2}\left(\dot{q}^{T} \tilde{\beta} q\right)^{2} \rightarrow 0\right\}$ or the invariant set $\left\{\left(q^{T}, \dot{q}^{T}\right)^{T} \in\right.$ $\mathbb{R}^{2 N}$, s.t. $\left.l^{-2}\left(\dot{q}^{T} \tilde{\beta} q\right)^{2} \leq \frac{1}{k_{1}}\right\}$ if $\frac{c}{2 k_{2}}>\frac{1}{k_{1}}$, and converges to the $\omega$-limit set $S_{2}=\left\{\left(q^{T}, \dot{q}^{T}\right)^{T} \in \mathbb{R}^{2 N}\right.$, s.t. $1-$ $\left.k_{1} l^{-2}\left(\dot{q}^{T} \tilde{\beta} q\right)^{2} \rightarrow 0\right\}$ or the invariant set $\left\{\left(q^{T}, \dot{q}^{T}\right)^{T} \in\right.$ $\mathbb{R}^{2 N}$, s.t. $\left.l^{-2}\left(\dot{q}^{T} \tilde{\beta} q\right)^{2} \leq \frac{c}{2 k_{2}}\right\}$ if $\frac{c}{2 k_{2}} \leq \frac{1}{k_{1}}$, where $c$ is such that $\dot{K}(t)<c I_{n \times n}, \forall t$.

Proof:

Let us consider again the time-varying Lyapunov function (18). Its derivative along the dynamics (15), with non-zero disturbance $F(t)$ writes as

$$
\begin{aligned}
& \dot{V}(z, t)=\dot{q}^{T}(-C \dot{q}-K q-\beta U q)+q^{T} K \dot{q} \\
& +q^{T} \dot{K}(t) q+\dot{q}^{T} F(t) \\
& =-\dot{q}^{T} C \dot{q}-\dot{q}^{T} \beta q U+q^{T} \dot{K}(t) q+\dot{q}^{T} F(t)
\end{aligned}
$$

under Assumption 1, we can write

$$
\dot{V}(z, t) \leq-\dot{q}^{T} \beta q U+\dot{q}^{T} F(t)+\frac{1}{2} c q^{2}
$$

which under Assumption 2, gives

$$
\leq-\dot{q}^{T} \beta q U+|\dot{q}| F_{\max }+\frac{1}{2} c q^{2}
$$

substituting $U$ by the controller (24), leads to

$$
\begin{gathered}
\leq-k_{1} l^{-2}\left(\dot{q}^{T} \tilde{\beta} q \mid\right)^{2} \epsilon|\dot{q}|+|\dot{q}| F_{\max }\left(1-k_{1} l^{-2}\left(\dot{q}^{T} \tilde{\beta} q\right)^{2}\right) \\
+\left|q^{2}\right|\left(\frac{c}{2}-k_{2} l^{-2}\left(\dot{q}^{T} \tilde{\beta} q\right)^{2}\right) \\
\leq|\dot{q}| F_{\max }\left(1-k_{1} l^{-2}\left(\dot{q}^{T} \tilde{\beta} q\right)^{2}\right) \\
+\left|q^{2}\right|\left(\frac{c}{2}-k_{2} l^{-2}\left(\dot{q}^{T} \tilde{\beta} q\right)^{2}\right)
\end{gathered}
$$

- Case 1: $\frac{c}{2 k_{2}}>\frac{1}{k_{1}}$

In this case as long as $\left(\dot{q}^{T} \tilde{\beta} q\right)^{2}>\frac{c}{2 k_{2}}>\frac{1}{k_{1}}$, then $\dot{V}<0$, which makes $x$ decreasing until it enters the invariant set $\left\{\left(q^{T}, \dot{q}^{T}\right)^{T} \in \mathbb{R}^{2 N}\right.$, s.t. $\left.l^{-2}\left(\dot{q}^{T} \tilde{\beta} q\right)^{2} \leq \frac{c}{2 k_{2}}\right\}$, which makes $V(z, t)$ bounded. Here we have to distinguish two cases: 1- First, the trajectories keep decreasing until they reach the invariant set

$$
\left\{\left(q^{T}, \dot{q}^{T}\right)^{T} \in \mathbb{R}^{2 N} \text {, s.t. } l^{-2}\left(\dot{q}^{T} \tilde{\beta} q\right)^{2} \leq \frac{1}{k_{1}}\right\}
$$

2- Second, the trajectories are stuck in the domain where $\left(\dot{q}^{T} \tilde{\beta} q\right)^{2} \leq \frac{c}{2 k_{2} l^{-2}}$ and $\left(\dot{q}^{T} \tilde{\beta} q\right)^{2}>\frac{1}{k_{1} l^{-2}}$. Since in this set we have $|\dot{q}| F_{\max }\left(1-k_{1} l^{-2}\left(\dot{q}^{T} \tilde{\beta} q\right)^{2}\right) \leq 0$, we can write

$$
\dot{V}(z, t) \leq\left|q^{2}\right|\left(\frac{c}{2}-k_{2} l^{-2}\left(\dot{q}^{T} \tilde{\beta} q\right)^{2}\right)
$$

which together with the boundedness of $V$ gives

$$
\begin{gathered}
+\infty>V(z(0), 0)-V(z(t), t) \\
\geq-\int_{0}^{t} q^{2}(t)\left(\frac{c}{2}-k_{2} l^{-2}\left(\dot{q}(t)^{T} \tilde{\beta} q(t)\right)^{2}\right) d t \\
\Rightarrow \int_{0}^{t} q^{2}(t)\left(\frac{c}{2}-k_{2} l^{-2}\left(\dot{q}(t)^{T} \tilde{\beta} q(t)\right)^{2}\right) d t \text { is bounded. }
\end{gathered}
$$

\begin{tabular}{|l|l|l|}
\hline Parameters & Definitions & Values \\
\hline$n$ & Number of ropes & $8[-]$ \\
$m_{e}$ & Mass of the car & $3500[\mathrm{~kg}]$ \\
$\rho$ & Main rope linear mass density & $2.11[\mathrm{~kg} / \mathrm{m}]$ \\
$l$ & Rope maximum length & $390[\mathrm{~m}]$ \\
$H$ & Building height & $402.8[\mathrm{~m}]$ \\
$c_{p}$ & Damping coefficient & $0.0315[\mathrm{N.sec} / \mathrm{m}]$ \\
\hline
\end{tabular}

Table 1. Numerical values of the mechanical parameters

Now, due to the boundedness of $V$ we conclude about the boundedness of $q, \dot{q}$, furthermore, using Assumption 1 and the system equations (15), we conclude about the boundedness of $\ddot{q}$. Boundedness of $\dot{q}$ and $\ddot{q}$ implies that $q^{2}(t)\left(\frac{c}{2}-\right.$ $\left.k_{2} l^{-2}\left(\dot{q}(t)^{T} \tilde{\beta} q(t)\right)^{2}\right)$ is uniform continuous. Finally, using Barbalat's Lemma we conclude that $\lim _{t \rightarrow \infty} q^{2}(t)\left(\frac{c}{2}-\right.$ $\left.k_{2} l^{-2}\left(\dot{q}(t)^{T} \tilde{\beta} q(t)\right)^{2}\right)=0$. Next, by examining the system equations (15), we can conclude that $\lim _{t \rightarrow \infty} q^{2}(t)=0$ cannot be a solution of (15), since there is no assumption on $F(t)$ converging to zero when $t \rightarrow 0$. Thus, we finally conclude that in this second case, the solution $q, \dot{q}$ satisfies $\lim _{t \rightarrow \infty} \frac{c}{2}-k_{2} l^{-2}\left(\dot{q}(t)^{T} \tilde{\beta} q(t)\right)^{2}=0$.

- Case 2: $\frac{c}{2 k_{2}} \leq \frac{1}{k_{1}}$ : Following the same reasoning as in case 1 , we can conclude that the solution $q, \dot{q}$ either converges to the invariant set $\left\{\left(q^{T}, \dot{q}^{T}\right)^{T} \in \mathbb{R}^{2 N}\right.$, s.t. $l^{-2}\left(\dot{q}^{T} \tilde{\beta} q\right)^{2} \leq$ $\left.\frac{c}{2 k_{2}}\right\}$ or satisfies $\lim _{t \rightarrow \infty} 1-k_{1} l^{-2}\left(\dot{q}^{T} \tilde{\beta} q\right)^{2}=0$.

Remark 1. The controllers (17), (24) are state feedbacks based on $q, \dot{q}$, these states can be easily computed from the sway measurements at $N$ given positions $y(1), \ldots, y(N)$, via equation (10). The sway $w(y, t)$ can be measured by laser displacement sensors placed at the positions $y(i), i=$ $1,2, \ldots N$, along the rope, e.g. Otsuki et al. [2002], subsequently $q$ can be computed by simple algebraic inversion of (10), and $\dot{q}$ can be obtained by direct numerical differentiation of $q$.

\section{NUMERICAL EXAMPLE}

The case of an elevator system with the mechanical parameters summarized on Table 1 has been considered for the tests presented hereafter. We write the controllers based on the model (15), (16) with one mode, but we test them on a model with three modes. The fact is that one mode is enough since when comparing the solution of the PDE (7) to the discrete model (15) the higher modes shown to be negligible, and a discrete model with one mode showed a very good match with the PDE model, but to make the simulation tests more realistic we chose to test the controllers on a three modes model. Furthermore, to make the simulation tests more challenging we added a random white noise to the states fed back to the controller (equivalent to about $\pm 1 \mathrm{~cm}$ of error on the rope sway measurement from which the states are computed, see Remark 1), and we filtered the control signal with a first order filter with a cut frequency of $10 \mathrm{hz}$ and a delay term of 5 sampling times, to simulate actuator dynamics and delays due to signal transmission and computation time. We first start with the validation of Theorem 1. The controller (17) has been implemented with $u_{\max }=1500 \mathrm{~N}$, starting the simulation with nonzero initial condition $q(0)=4, \dot{q}(0)=1$, and with zero external disturbances $f_{1}(t)=f_{2}(t)=0$. We also fixed the damping coefficient $c_{p}$ to zero, to see the damping effect of the controller alone. Following W.D.Zhu and Chen [2006], the tested time-varying rope length is depicted on 


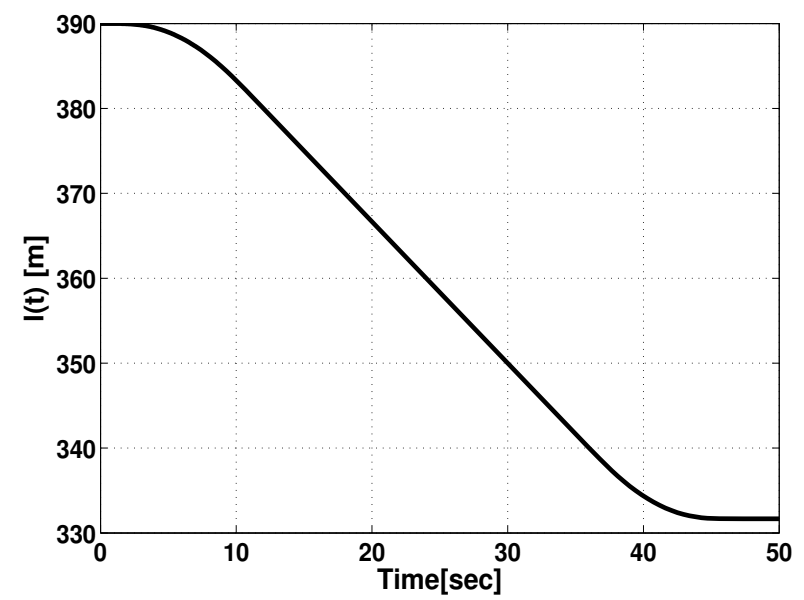

Fig. 2. Time-varying rope length

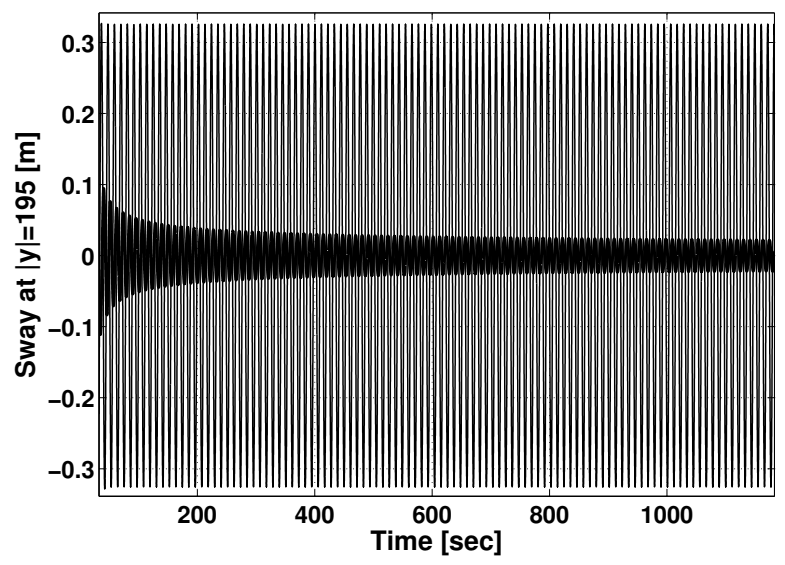

Fig. 3. Rope sway at $y=195 \mathrm{~m}$ : No control (thin line)With controller (17) (bold line)

Figures 2. The sway signal is depicted on Figures ${ }^{1} 3$ and 4 , where both the controlled and the uncontrolled sway signals are reported. The corresponding control signal is reported on Figures 5, and 6. These numerical results are in concordance with the asymptotic convergence results of Theorem 1.

Eventually, we report the numerical results corresponding to Theorem 2. We tested the controller (24), with the gains: $u_{\max }=150, F_{\max }=1, k_{1}=3000, k_{2}=15, \epsilon=$ 0.1 . Figures 7 , and 8 , show the sway without control vs. the sway with control at half-rope length. The effect of the controller (24) is clear, i.e., the maximum sway in transient phase is reduced from $0.8 \mathrm{~m}$ to about $0.3 \mathrm{~m}$, and the steady state sway is reduced by half. The corresponding continuous control signal is shown on Figures 9 and 10.

\section{CONCLUSION}

In this paper we have studied the problem of active control of elevator rope sway dynamics occurring due to external force disturbances acting on the elevator system with timevarying rope lengths. We have selected one actuation configuration, namely, a force actuator placed at the bottom of the elevator shaft pulling on the compensation sheave.

1 The figures' zoom is included for the reader to have a better idea about the signals shape.

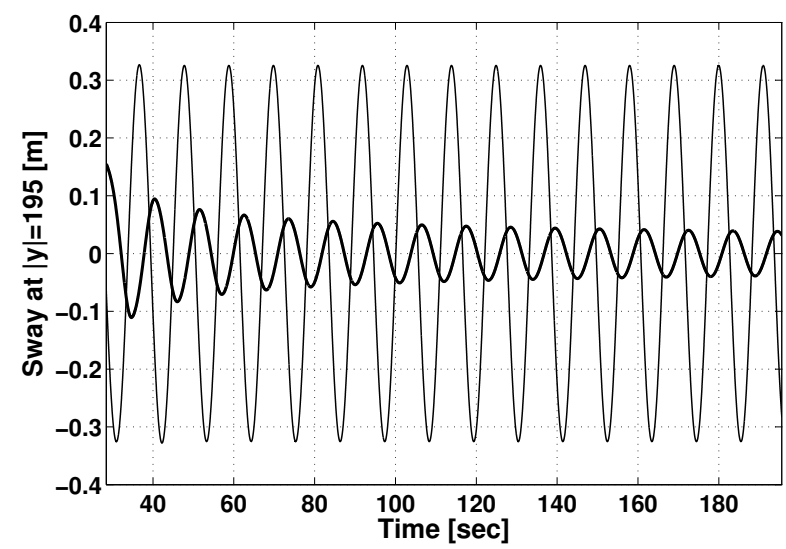

Fig. 4. Zoom of rope sway at $y=195 \mathrm{~m}$ : No control (thin line)- With controller (17) (bold line)

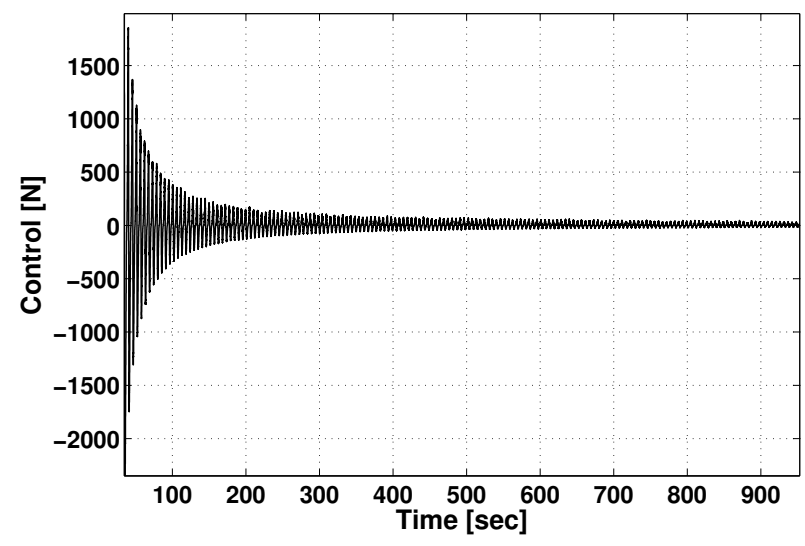

Fig. 5. Output of controller (17)

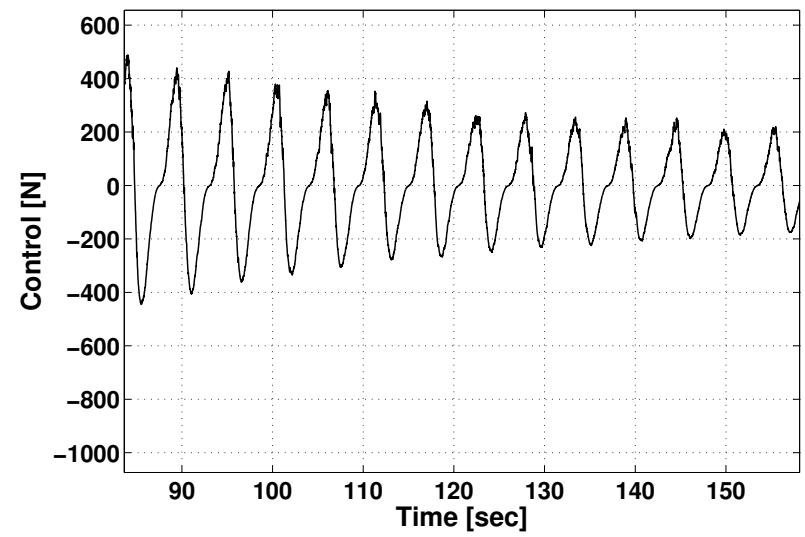

Fig. 6. Output of controller (17)- Zoom

For the selected actuation configuration, we have proposed nonlinear controllers based on Lyapunov theory. We have presented the stability analysis of these controllers and shown their efficiency using numerical tests. The numerical results reported here shown a very good performance of the proposed controllers when using a force actuator pulling on the ropes via the compensation sheave. However, other actuation methods might be feasible, therefore, one future research direction is to compare on the same test-case the 


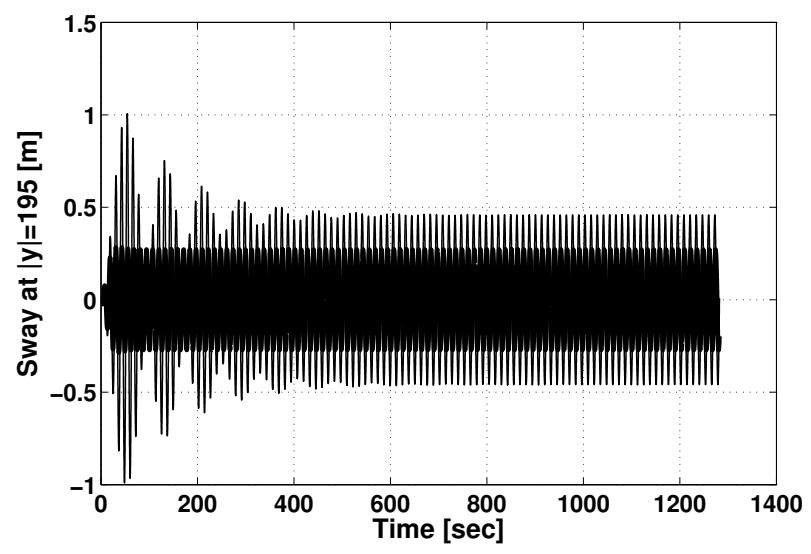

Fig. 7. Rope sway at $y=195 \mathrm{~m}$ : No control (thin line)With controller (24) (bold line)

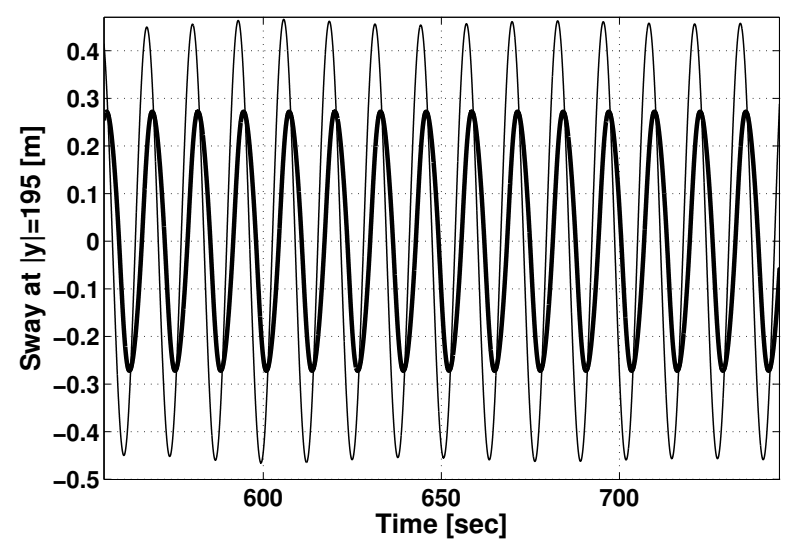

Fig. 8. Zoom of $\mathrm{r}$ sway at $y=195 \mathrm{~m}$ : No control (thin line)- With controller (24) (bold line)

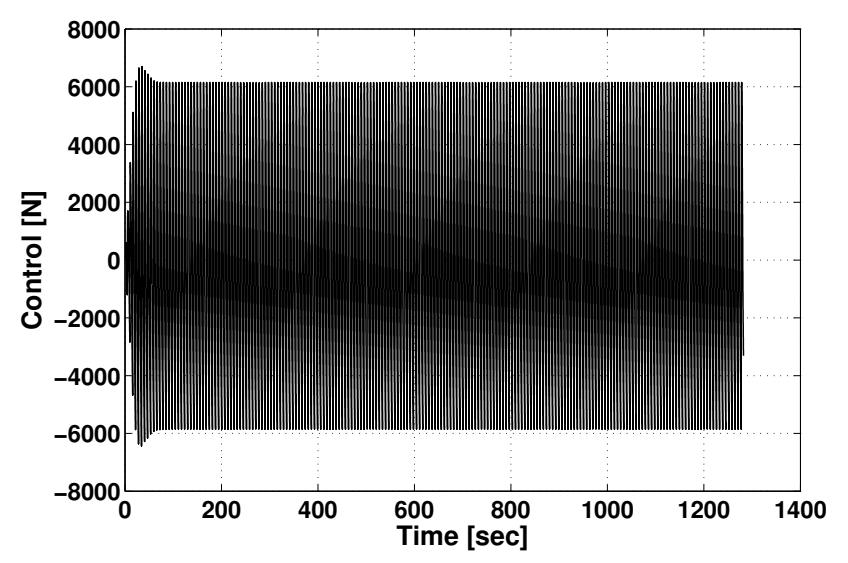

Fig. 9. Output of controller (24)

performance of different controllers designed for different actuation configurations.

\section{REFERENCES}

M. Benosman and D. Fukui. Lyapunov-based control of the sway dynamics for elevator ropes. 2014. Submitted to IEEE American Control Conference 2014.

Y. Fujino, P. Warnitchai, and B.M. Pacheco. Active stiffness control of cable vibration. ASME Journal of Applied Mechanics, 60:948-953, 1993.

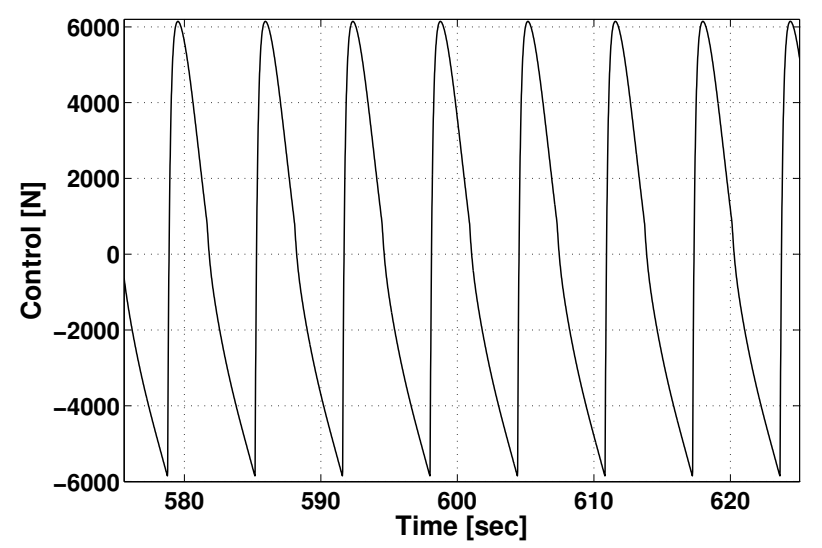

Fig. 10. Output of controller (24)- Zoom

S. Kaczmarczyk. Nonlinear sway and active stiffness control of long moving ropes in high-rise vertical transporation systems. In Springer Proceedings in Physics, editor, The 10th International Conference on Vibration Problems, pages 183-188, 2011.

S. Kaczmarczyk, R. Iwankiewicz, and Y. Terumichi. The dynamic behavior of a non-stationary elevator compensating rope system under harmonic and stochastic excitations. In Journal of Physics: Conference Series 181, pages 1-8. IOP Publishing, 2009.

H.K. Khalil. Nonlinear systems. New York Macmillan, second edition, 1996.

L. Meirovitch. Analytical methods in vibrations. Applied Mechanics. Collier Macmillan publishers, Fred Landis edition, 1967.

M. Otsuki, K. Yoshida, K. Nagata, S. Fujimoto, and T. Nakagawa. Experimental study on vibration control for rope-sway of elevator of high-rise building. In American Control Conference, pages 238-243, 2002.

W.D.Zhu and Y. Chen. Theoretical and experimental investigation of elevator cable dynamics and control. Journal of Sound and Vibration, 128:66-78, 2006.

W.D.Zhu and L.J Teppo. Design and analysis of a scaled model of a high-rise high-speed elevator. Journal of Sound and Vibration, 264:707-731, 2003.

W.D.Zhu and G.Y. Xu. Vibration of elevator cables with small bending stiffness. Journal of Sound and Vibration, 263:679-699, 2003. 